PROCEEDINGS OF THE

AMERICAN MATHEMATICAL SOCIETY

Volume 128, Number 6, Pages 1691-1695

S 0002-9939(99)05262-4

Article electronically published on September 30, 1999

\title{
THE FURUTA INEQUALITY IN BANACH $*$-ALGEBRAS
}

\author{
KÔTARÔ TANAHASHI AND ATSUSHI UCHIYAMA
}

(Communicated by David R. Larson)

\begin{abstract}
Let $0<p, q, r \in \mathbb{R}$ be real numbers with $p+2 r \leq(1+2 r) q$ and $1 \leq q$. Furuta (1987) proved that if bounded linear operators $A, B \in B(H)$ on a Hilbert space $H$ satisfy $O \leq B \leq A$, then $B^{\frac{p+2 r}{q}} \leq\left(B^{r} A^{p} B^{r}\right)^{\frac{1}{q}}$. This inequality is called the Furuta inequality and has many applications. In this paper, we prove that the Furuta inequality holds in a unital hermitian Banach *-algebra with continuous involution.
\end{abstract}

Let $A, B$ be bounded linear operators on a Hilbert space $H$. The celebrated Löwner-Heinz inequality states the following;

Theorem A (Löwner-Heinz inequality 4], [5]). Let $A, B \in B(H)$ satisfy $O \leq B$ $\leq A$. If $0<p<1$, then $B^{p} \leq A^{p}$.

For an extension of Theorem A, Furuta obtained the following interesting inequality in [1] and one page elementary proof in [2].

Theorem B (Furuta inequality [1, 2]). Let $0 \leq p, q, r \in \mathbb{R}$ and $A, B \in B(H)$ satisfy $O \leq B \leq A$. If $p+2 r \leq(1+2 r) q$ and $1 \leq q$ as shown in the figure, then $B^{\frac{p+2 r}{q}} \leq\left(B^{r} A^{p} B^{r}\right)^{\frac{1}{q}}$

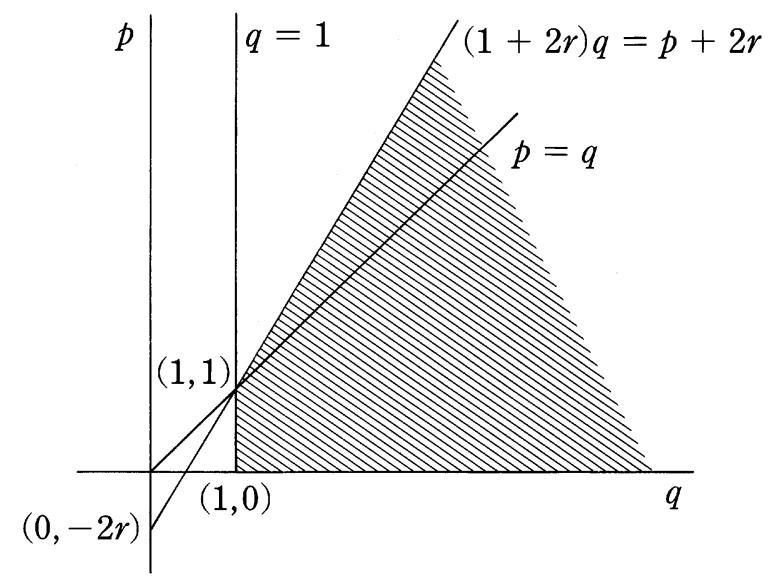

Received by the editors February 12, 1998 and, in revised form, July 13, 1998.

1991 Mathematics Subject Classification. Primary 47A05, 47B15.

Key words and phrases. The Löwner-Heinz inequality, the Furuta inequality.

This research is partially supported by Grant-in-Aid Scientific Research (K. Tanahashi, No. 10640185). 
One of the authors, Tanahashi [8, proved that the domain drown for $p, q$ and $r$ in Figure is the best possible one for Theorem B. Many applications of Theorem B have been developed, but we omit citing them.

Let $A$ be a unital Banach $*$-algebra with unit $e$ and $a, b \in A$. $A$ is called hermitian if $a=a^{*}$ implies $\sigma(a) \subset \mathbb{R}$ where $\sigma(a)$ denotes the spectrum of $a . a \geq 0$ means that $a=a^{*}$ and $\sigma(a) \subset[0, \infty) . a>0$ means $a \geq 0$ and $0 \notin \sigma(a) . a \geq b$ means $a-b \geq 0$, and $a>b$ means $a-b>0$. Let $\alpha \in \mathbb{R}$. For $a>0, a^{\alpha}$ means $\exp (\alpha \log a)$ where $\log$ is the principal branch of the complex logarithm. By the Shirali-Ford theorem [7], $\sigma\left(a^{*} a\right) \subset[0, \infty)$ for all $a \in A$.

Recently, Okayasu [6] proved that the Löwner-Heinz inequality holds in a unital hermitian Banach $*$-algebra with continuous involution.

Theorem C ([6]). Let $A$ be a unital hermitian Banach $*$-algebra with continuous involution. Let $a, b \in A$ and $p \in(0,1]$. Then $a^{p}<b^{p}$ if $0<a<b$, and $a^{p} \leq b^{p}$ if $0<a \leq b$.

In this paper, we prove that the Furuta inequality holds in this case.

Theorem 1. Let $A$ be a unital hermitian Banach $*$-algebra with continuous involution and $a, b \in A$. Let $0 \leq p, q, r \in \mathbb{R}$ satisfy $p+2 r \leq(1+2 r) q$ and $1 \leq q$. Then

$$
b^{\frac{p+2 r}{q}}<\left(b^{r} a^{p} b^{r}\right)^{\frac{1}{q}} \quad \text { if } \quad 0<b<a,
$$

and

$$
b^{\frac{p+2 r}{q}} \leq\left(b^{r} a^{p} b^{r}\right)^{\frac{1}{q}} \quad \text { if } \quad 0<b \leq a .
$$

To prove Theorem 1, we need following lemmas.

Lemma 2. If $0<a$, then $0<a^{-1}$.

Proof. Let $0<a$. Then $\sigma(a) \subset(0, \infty)$, and $\sigma\left(a^{-1}\right)=\sigma(a)^{-1} \subset(0, \infty)$. This implies $0<a^{-1}$.

Lemma 3. If $0<a<e$, then $e<a^{-1}$. Also, if $0<a \leq e$, then $e \leq a^{-1}$.

Proof. Let $0<a<e$. Then

$$
a^{-1}-e=a^{-\frac{1}{2}}(e-a) a^{-\frac{1}{2}}=\left\{(e-a)^{\frac{1}{2}} a^{-\frac{1}{2}}\right\}^{*}\left\{(e-a)^{\frac{1}{2}} a^{-\frac{1}{2}}\right\} .
$$

This implies $0<a^{-1}-e$ and $e<a^{-1}$. The rest of the proof is similar.

Lemma 4. If $0<b<a$, then $0<a^{-1}<b^{-1}$. Also, if $0<b \leq a$, then $0<a^{-1} \leq$ $b^{-1}$.

Proof. Let $0<b<a$. Then

$$
a^{-\frac{1}{2}} b a^{-\frac{1}{2}}=\left(b^{\frac{1}{2}} a^{-\frac{1}{2}}\right)^{*}\left(b^{\frac{1}{2}} a^{-\frac{1}{2}}\right)
$$

and

$$
e-a^{-\frac{1}{2}} b a^{-\frac{1}{2}}=a^{\frac{1}{2}}(a-b) a^{-\frac{1}{2}}=\left\{(a-b)^{\frac{1}{2}} a^{-\frac{1}{2}}\right\}^{*}\left\{(a-b)^{\frac{1}{2}} a^{-\frac{1}{2}}\right\} .
$$

This implies $0<a^{-\frac{1}{2}} b a^{-\frac{1}{2}}<e$. Hence, by Lemma 3 ,

$$
e<\left(a^{-\frac{1}{2}} b a^{-\frac{1}{2}}\right)^{-1}=a^{\frac{1}{2}} b^{-1} a^{\frac{1}{2}} .
$$


Then

$$
\begin{aligned}
b^{-1}-a^{-1} & =a^{-\frac{1}{2}}\left(a^{\frac{1}{2}} b^{-1} a^{\frac{1}{2}}-e\right) a^{-\frac{1}{2}} \\
& =\left\{\left(a^{\frac{1}{2}} b^{-1} a^{\frac{1}{2}}-e\right) a^{-\frac{1}{2}}\right\}^{*}\left\{\left(a^{\frac{1}{2}} b^{-1} a^{\frac{1}{2}}-e\right) a^{-\frac{1}{2}}\right\} .
\end{aligned}
$$

This impies $0<b^{-1}-a^{-1}$. Hence $0<a^{-1}<b^{-1}$ by Lemma 2 .

Let $0<b \leq a$. Then $0<a$ by Lemma 2 of [6]. The rest of the proof is similar.

Lemma 5. If $0<b<a$ and $0<c$, then $c b c<c a c$. Also, if $0<b \leq a$ and $0<c$, then $c b c \leq c a c$.

Proof. Let $0<b<a$ and $0<c$. Then

$$
c a c-c b c=c(a-b) c=\left\{(a-b)^{\frac{1}{2}} c\right\}^{*}\left\{(a-b)^{\frac{1}{2}} c\right\} .
$$

This implies $0<c a c-c b c$ and $c b c<c a c$. The rest of the proof is similar.

Lemma 6. If $0<a$, then $\left(a^{2}\right)^{\frac{1}{2}}=a$.

Proof. Since $\sigma\left(a^{2}\right)=\{\sigma(a)\}^{2}$, there exists a positively oriented circle $\Lambda$ containing $\sigma\left(a^{2}\right)$ with $0<\Re \lambda$ for all $\lambda \in \Lambda$. Let

$$
\lambda=|\lambda| e^{i \theta} \in \Lambda \quad \text { with } \quad-\frac{\pi}{2}<\theta<\frac{\pi}{2},
$$

and

$$
\Gamma=\left\{\mu=|\lambda|^{\frac{1}{2}} e^{\frac{1}{2} i \theta} \quad|\quad \lambda=| \lambda \mid e^{i \theta} \in \Lambda\right\} .
$$

Then

$$
\begin{aligned}
\log a^{2} & =\int_{\Lambda}(\log \lambda)\left(\lambda-a^{2}\right)^{-1} d \lambda \\
& =\int_{\Gamma}\left(\log \mu^{2}\right)\left(\mu^{2}-a^{2}\right)^{-1} 2 \mu d \mu \\
& =\int_{\Gamma} 2(\log \mu)(\mu+a)^{-1} d \mu+\int_{\Gamma} 2(\log \mu)(\mu-a)^{-1} d \mu \\
& =0+2 \log a=2 \log a .
\end{aligned}
$$

Thus

$$
\left(a^{2}\right)^{\frac{1}{2}}=e^{\frac{1}{2} \log a^{2}}=e^{\log a}=a .
$$

Lemma 7. If $0<a, b$ and $\lambda$ is a real number, then

$$
(b a b)^{\lambda}=b a^{\frac{1}{2}}\left(a^{\frac{1}{2}} b^{2} a^{\frac{1}{2}}\right)^{\lambda-1} a^{\frac{1}{2}} b .
$$

Proof. Let

$$
F(\lambda)=(b a b)^{\lambda} \quad \text { and } \quad G(\lambda)=b a^{\frac{1}{2}}\left(a^{\frac{1}{2}} b^{2} a^{\frac{1}{2}}\right)^{\lambda-1} a^{\frac{1}{2}} b
$$


Since $b a b=\left(a^{\frac{1}{2}} b\right)^{*}\left(a^{\frac{1}{2}} b\right)$, we have $0<F(\lambda), G(\lambda)$. By the definitions, we have $F(1)=G(1)$. Also,

$$
\begin{aligned}
\left\{G\left(2^{-1}\right)\right\}^{2} & =\left\{b a^{\frac{1}{2}}\left(a^{\frac{1}{2}} b^{2} a^{\frac{1}{2}}\right)^{-\frac{1}{2}} a^{\frac{1}{2}} b\right\}\left\{b a^{\frac{1}{2}}\left(a^{\frac{1}{2}} b^{2} a^{\frac{1}{2}}\right)^{-\frac{1}{2}} a^{\frac{1}{2}} b\right\} \\
& =b a^{\frac{1}{2}}\left(a^{\frac{1}{2}} b^{2} a^{\frac{1}{2}}\right)^{-\frac{1}{2}} a^{\frac{1}{2}} b^{2} a^{\frac{1}{2}}\left(a^{\frac{1}{2}} b^{2} a^{\frac{1}{2}}\right)^{-\frac{1}{2}} a^{\frac{1}{2}} b \\
& =b a b=\left\{F\left(2^{-1}\right)\right\}^{2} .
\end{aligned}
$$

Hence $F\left(2^{-1}\right)=G\left(2^{-1}\right)$ by Lemma 6 . Similarly, we have

$$
F\left(2^{-n}\right)=G\left(2^{-n}\right) \quad \text { for } \quad n=2,3, \cdots .
$$

Since $F(\lambda), G(\lambda)$ are analytic on the real line $\mathbb{R}$ and $2^{-n} \rightarrow 0(n \rightarrow \infty)$, we have

$$
F(\lambda)=G(\lambda) \quad \text { for } \quad \lambda \in \mathbb{R} .
$$

Remark. We remark that Lemma 7 has been obtained in [3] for operator case on Hilbert space, but our method in Banach *-algebra case is completely different from one in 3 .

Proof of Theorem 1. Let $0<b<a$. We show first the case $0<p \leq 1$. In this case, $b^{p}<a^{p}$ by Theorem C, and $b^{p+2 r}<b^{r} a^{p} b^{r}$ by Lemma 5 . Hence $b^{\frac{p+2 r}{q}}<\left(b^{r} a^{p} b^{r}\right)^{\frac{1}{q}}$ by Theorem C. We consider the case $1<p$. We may assume $q=\frac{p+2 r}{1+2 r}$ by Theorem C. Let $0<r \leq \frac{1}{2}$. Then $0<b^{2 r}<a^{2 r}$ by Theorem C. Hence

$$
\begin{aligned}
b^{-r}\left(b^{r} a^{p} b^{r}\right)^{\frac{1}{q}} b^{-r} & =b^{-r} b^{r} a^{\frac{p}{2}}\left(a^{\frac{p}{2}} b^{2 r} a^{\frac{p}{2}}\right)^{\frac{1}{q}-1} a^{\frac{p}{2}} b^{r} b^{-r} \\
& =a^{\frac{p}{2}}\left(a^{\frac{p}{2}} b^{2 r} a^{\frac{p}{2}}\right)^{\frac{1-p}{p+2 r}} a^{\frac{p}{2}}
\end{aligned}
$$

by Lemma 7 . Since $a^{\frac{p}{2}} b^{2 r} a^{\frac{p}{2}}<a^{p+2 r}$ by Lemma 5 and $-1<\frac{1-p}{p+2 r}<0$, we have

$$
b^{-r}\left(b^{r} a^{p} b^{r}\right)^{\frac{1}{q}} b^{-r}>a^{\frac{p}{2}}\left(a^{p+2 r}\right)^{\frac{1-p}{p+2 r}} a^{\frac{p}{2}}=a>b
$$

by Lemma 4 and Theorem C. Hence

$$
\left(b^{r} a^{p} b^{r}\right)^{\frac{1}{q}}>b^{1+2 r} \quad \text { for } q=\frac{p+2 r}{1+2 r}
$$

by Lemma 5 . The rest of the proof is the same as in [1], but we cite its proof for the sake of importance as follows.

Put $a_{1}=\left(b^{r} a^{p} b^{r}\right)^{\frac{1}{q}}$ and $b_{1}=b^{1+2 r}$ in (1). Repeating (1) again for $a_{1}>b_{1}>$ $0,0<r_{1} \leq \frac{1}{2}$ and $1<p_{1}$,

$$
\left(b_{1}^{r_{1}} a_{1}^{p_{1}} b_{1}^{r_{1}}\right)^{\frac{1}{q_{1}}}>b_{1}^{1+2 r_{1}} \quad \text { for } \quad q_{1}=\frac{p_{1}+2 r_{1}}{1+2 r_{1}} .
$$

Put $p_{1}=q \geq 1$ and $r_{1}=\frac{1}{2}$; then

$$
\left(b^{2 r+\frac{1}{2}} a^{p} b^{2 r+\frac{1}{2}}\right)^{\frac{1}{q_{1}}}>b^{2(1+2 r)} .
$$


Put $s=2 r+\frac{1}{2}$. Then $q_{1}=\frac{p_{1}+2 r_{1}}{1+2 r_{1}}=\frac{p+2 s}{1+2 s}$ since $p_{1}=q$ and $2(1+2 r)=1+2 s$. Hence

$$
\left(b^{s} a^{p} b^{s}\right)^{\frac{1}{q_{1}}}>b^{1+2 s} \quad \text { for } \quad q_{1}=\frac{p+2 s}{1+2 s} .
$$

Since $r \in\left(0, \frac{1}{2}\right]$ in (1), $s=2 r+\frac{1}{2} \in\left(\frac{1}{2}, \frac{3}{2}\right]$ in (2). Consequently (1) holds for $r \in\left(0, \frac{3}{2}\right]$ and $q=\frac{p+2 r}{1+2 r}$. Repeating this method, (1) holds for each $r>0$ and $q=\frac{p+2 r}{1+2 r}$.

If $0<b \leq a$, then $a$ is invertible by Lemma 2 of $[6]$ and $0<a^{-1} \leq b^{-1}$. The rest of the proof is similar to the case $0<b<a$.

\section{ACKNOWLEDGEMENTS}

The authors would like to express their thanks to Professor T. Okayasu and the referee for heart-warming suggestions.

\section{REFERENCES}

[1] T. Furuta, $A \geq B \geq O$ assures $\left(B^{r} A^{p} B^{r}\right)^{\frac{1}{q}} \geq B^{\frac{p+2 r}{q}}$ for $r \geq 0, p \geq 0, q \geq 1$ with $(1+2 r) q \geq(p+2 r)$, Proc. Amer. Math. Soc., 101 (1987), 85-88. MR 89b:47028

[2] T. Furuta, An elementary proof of an order preserving inequality, Proc. Japan Acad., 65 (1989), 126. MR 90g:47029

[3] T. Furuta, Two operator functions with monotone property, Proc. Japan Acad., 111 (1991), 511-516. MR 91f:47023

[4] E. Heinz, Beiträge zur Störungstheorie der Spektralzerlegung, Math. Ann., 123 (1951), 415-438. MR 13:471f

[5] K. Löwner, Über monotone Matrixfunktionen, Math. Z., 38 (1934), 177-216.

[6] T. Okayasu, Heinz's inequality in Banach *-algebras, ( preprint ).

[7] S. Shirali and J. W. M. Ford, Symmetry in complex involutory Banach algebras II, Duke Math. J., 37 (1970), 275-280. MR 41:5977

[8] K. Tanahashi, Best possibility of the Furuta inequality, Proc. Amer. Math. Soc., 124 (1996), 141-146. MR 96d:47025

Department of Mathematics, Tohoku College of Pharmacy, Komatsushima, Aoba-ku, SENDAI 981-8558, JAPAN

Mathematical Institute, Tohoku University, Aoba-ku, Sendai 980-8578, Japan 